\title{
A Direct Fusion Drive for Rocket Propulsion
}

\author{
Yosef S. Razinn, ${ }^{\mathrm{a}, *}$, Gary Pajer ${ }^{\mathrm{a}}$, Mary Breton ${ }^{\mathrm{a}}$, Eric Hamª ${ }^{\mathrm{a}}$ Joseph Mueller ${ }^{\mathrm{a}}$, Michael Paluszek ${ }^{\mathrm{a}}$, Alan H. Glasser ${ }^{\mathrm{b}}$, \\ Samuel A. Cohen ${ }^{c}$ \\ ${ }^{a}$ Princeton Satellite Systems, 6 Market St, Suite 926, Plainsboro, NJ, USA, 08536-2096 \\ ${ }^{b}$ Department of Aeronautics $\mathcal{F}$ Astronautics, University of Washington, Box 352400, Seattle, WA, USA, 98195-2400 \\ ${ }^{c}$ Princeton Plasma Physics Laboratory, P.O. Box 451, Princeton, NJ, USA, 08543-0451
}

\begin{abstract}
The Direct Fusion Drive (DFD), a compact, anuetronic fusion engine, will enable more challenging exploration missions in the solar system. The engine proposed here uses a deuterium-helium-3 reaction to produce fusion energy by employing a novel field-reversed configuration (FRC) for magnetic confinement. The FRC has a simple linear solenoid coil geometry yet generates higher plasma pressure, hence higher fusion power density, for a given magnetic field strength than other magnetic-confinement plasma devices. Waste heat generated from the plasma's Bremsstrahlung and synchrotron radiation is recycled to maintain the fusion temperature. The charged reaction products, augmented by additional propellant, are exhausted through a magnetic nozzle. A 1 MW DFD is presented in the context of a mission to deploy the James Webb Space Telescope (6200 kg) from GPS orbit to a Sun-Earth L2 halo orbit in 37 days using just $353 \mathrm{~kg}$ of propellant and about half a kilogram of ${ }^{3} \mathrm{He}$. The engine is designed to produce $40 \mathrm{~N}$ of thrust with an exhaust velocity of $56.5 \mathrm{~km} / \mathrm{s}$ and has a specific power of $0.18 \mathrm{~kW} / \mathrm{kg}$.
\end{abstract}

Keywords: fusion, propulsion, FRC, deuterium, helium-3, aneutronic

\section{INTRODUCTION}

The future of space exploration, from robotic deep-space expeditions to manned interplanetary missions, will require high-thrust, high-exhaust velocity engines. These specifications shorten the transit time and reduce the mass of the spacecraft. Thus, operational costs, which for deep space missions can approach \$50M USD per year, are lowered. For manned missions, the decreased transit time has the additional benefit of reducing the astronauts' exposure to cosmic radiation and zero gravity conditions. Furthermore, on such missions, high-power propulsion is essential in the case of an aborted operation, as it would allow the astronauts to quickly return to Earth in case of an accident or emergency. The exact engine specifications, particularly its power and specific impulse, will vary for each mission. Thrust can be further augmented by the injection of additional propellant and the use of multiple engines. Herein, one such mission is illustrated powered by the Direct Fusion Drive engine (DFD) with moderate power $(\approx 1 \mathrm{MW})$ and specific impulse of at least $5,750 \mathrm{~s}$.

Many proposed NASA and ESA missions require high-performance propulsions systems, as shown in Table 1. All of these power requirements are exceeded by the output of the DFD, which can potentially achieve up to $20 \mathrm{MW}$ of power. Thus, the engine theoretically meets the anticipated requirements for deep-space and interplanetary manned missions. The $1 \mathrm{MW}$ DFD will be presented in the context of a mission to deploy the James Webb Space Telescope from a GPS orbit to a Sun-Earth L2 halo orbit.

Most other nuclear propulsion schemes, both fusion- and fission-based, only are realizable at power levels of GWs, at which point the mass of fuel, propellant, structure, and shielding severely limit their space flight capabilities.

\footnotetext{
*Principle Corresponding Author. Phone: (609).275.9606, Email: yrazin@psatellite.com

Email addresses: gpajer@psatellite.com (Gary Pajer), mbreton@mit.edu (Mary Breton), eham@psatellite.edu (Eric Ham), jmueller@psatellite.com (Joseph Mueller), map@psatellite.com (Michael Paluszek), ahg5@u . washington. edu (Alan H. Glasser), scohen@pppl.gov (Samuel A. Cohen)
} 
Table 1: Proposed NASA and ESA missions requiring high-power propulsion systems. The power was determined from the specific mass of the power sources.

\begin{tabular}{lllll}
\hline Mission & Power $(\mathrm{kW})$ & Power Source & Engine & References \\
\hline JIMO & 180 & Nuclear Fission & Nexis Ion \& Hall Thrusters & {$[52]$} \\
Outer Planets & 95 & Nuclear Fission & Nexis Ion & {$[52]$} \\
$200 \mathrm{AU}$ & 65 & Nuclear Fission & DS4G & {$[11]$} \\
$200 \mathrm{AU}$ & 160 & Solar Panels & Ion & {$[11]$} \\
NEO 2004 MN4 & 210 & Solar Panels & Ion & {$[32]$} \\
NEO Crew & 350 & Solar Panels & Hall & {$[32]$} \\
\hline
\end{tabular}

Nuclear thermal rockets, which have been demonstrated, prove very undesirable in comparison to the DFD. Their exhaust velocity is only $7.5 \mathrm{~km} / \mathrm{s}$, a fraction of direct fusion rockets [5]. In addition, all fission rockets take great risk by putting radioactive materials such as uranium and plutonium into orbit, which if a breach occurs could contaminate large swaths of the planet. Thus, fission engines face two overwhelming challenges: a potential for catastrophic failure and strong negative public sentiment about taking such risks at all. Fission electric schemes are limited in power conversion efficiency by the maximum temperature of the heat exchanger leading to low specific powers for the propulsion system [5]. Finally advanced fission rockets, such as nuclear pulse, gas core and nuclear light bulb, are even more theoretical than fusion- relying on technologies or materials that have yet to be developed.

Some specific advantages of a deuterium-helium-3 fueled (D- ${ }^{3} \mathrm{He}$ ) aneutronic fusion engine with thrust augmentation (i.e. the DFD) can be seen in Table 2, where it is compared against various engine configurations, including some conceptual designs. The electric propulsion options, which accelerate ions using electric fields with or without magnetic fields, require a separate power source and therefore the mass ratios given are underestimates. One electric propulsion system, the Dual-Stage 4-Grid system (DS4G), shows promise and analysis suggests performance similar to the fusion engine described in [12] and [11]. D- ${ }^{3} \mathrm{He}$ fusion without thrust augmentation supplies too little thrust, making it impractical for most missions. Adding propellant significantly slows down the fusion products and raises the thrust to a more effective level for space travel.

Table 2: Comparison of propulsion technologies for deploying the James Webb Space Telescope.

A $\Delta u$ of $3.1 \mathrm{~km} / \mathrm{s}$, required for a low thrust transfer from GPS orbit to L2, is used as a baseline for comparison. The mass ratios are calculated using the rocket equation, using the initial mass $m_{\mathrm{i}}$, final mass $m_{\mathrm{f}}$, exhaust velocity $u_{\mathrm{e}}$, and the total mission velocity change $\Delta u$. For the electric propulsion options, the energy source and its fuel are not specified as it is external to the propulsion system, therefore an advanced solar cell power system with specific power of $500 \mathrm{~W} / \mathrm{kg}$ [26] is used for the most favorable comparison. Fusion propulsion usually does not involve direct propulsion, but uses the fusion engine as a power source for heating, thus thrust data is not included for $\mathrm{D}-\mathrm{T}$ or $\mathrm{p}-{ }^{11} \mathrm{~B}$. The values for $\mathrm{D}-{ }^{3} \mathrm{He}$ are based on the results from the example mission in Section 4.

\begin{tabular}{|c|c|c|c|c|c|c|c|}
\hline Type & Fuel & Propellant & $\begin{array}{l}\text { Exhaust } \\
\text { Velocity, } \\
u_{\mathrm{e}}(\mathrm{km} / \mathrm{s})\end{array}$ & $\begin{array}{l}\text { Mass Ra- } \\
\text { tio, } m_{\mathrm{i}} / m_{\mathrm{f}} \\
(\mathrm{kg} / \mathrm{kg})\end{array}$ & $\begin{array}{l}\text { Thrust, } \\
\mathrm{T}(\mathrm{N})\end{array}$ & $\begin{array}{l}\text { Thrust- } \\
\text { to-weight } \\
\text { ratio }\end{array}$ & References \\
\hline Chemical (RL-10) & $\mathrm{LOx}_{-} \mathrm{LH}_{2}$ & $\mathrm{H}_{2} \mathrm{O}$ & 4.6 & 1.96 & $1.1 \times 10^{5}$ & 60.53 & {$[1,51]$} \\
\hline Ion (typical) & & $\mathrm{Xe}$ & 30 & 1.11 & 0.24 & $4.6 \times 10^{-4}$ & {$[40,50]$} \\
\hline Ion (DS4G) & & $\mathrm{Xe}$ & 140 & 1.02 & 1.0 & $5.1 \times 10^{-4}$ & [11] \\
\hline Hall & & $\mathrm{Ar}$ & 20 & 1.17 & 1.1 & $1.6 \times 10^{-3}$ & {$[13,46]$} \\
\hline MPD & & $\mathrm{Ar}$ & 27 & 1.12 & 12 & $7.1 \times 10^{-4}$ & {$[4,41]$} \\
\hline Fission & $\mathrm{U}, \mathrm{Pu}$ & $\mathrm{H}_{2}$ & 7.0 & 1.56 & $3.3 \times 10^{5}$ & 30 & {$[49,53]$} \\
\hline Nuclear Lightbulb & ${ }^{233} \mathrm{U}$ & $\mathrm{H}_{2}$ & 18 & 1.19 & $4.1 \times 10^{5}$ & 1.31 & {$[18,37]$} \\
\hline VASMIR & & $\mathrm{Ar}$ & 49 & 1.07 & 5 & $9.3 \times 10^{-5}$ & [3] \\
\hline Fusion & $\mathrm{D}-\mathrm{T}$ & ${ }^{4} \mathrm{He}$ & $1.3 \times 10^{4}$ & 1.00 & & & \\
\hline Fusion & $\mathrm{p}-{ }^{11} \mathrm{~B}$ & ${ }^{4} \mathrm{He}$ & $1.2 \times 10^{4}$ & 1.00 & & & \\
\hline Fusion & $\mathrm{D}-{ }^{3} \mathrm{He}$ & ${ }^{4} \mathrm{He}+\mathrm{p}$ & $2.5 \times 10^{4}$ & 1.00 & 0.054 & & \\
\hline$\overline{\mathrm{D}} \overline{\mathrm{F}} \overline{\mathrm{D}}$ & $\mathrm{D}^{-}{ }^{-3} \mathrm{He}^{-}$ & $\overline{\mathrm{D}}$ & $5 \overline{6}$ & 1.06 & $\overline{4} \overline{0}$ & $6 . \overline{5} \times 10^{-3}$ & \\
\hline
\end{tabular}




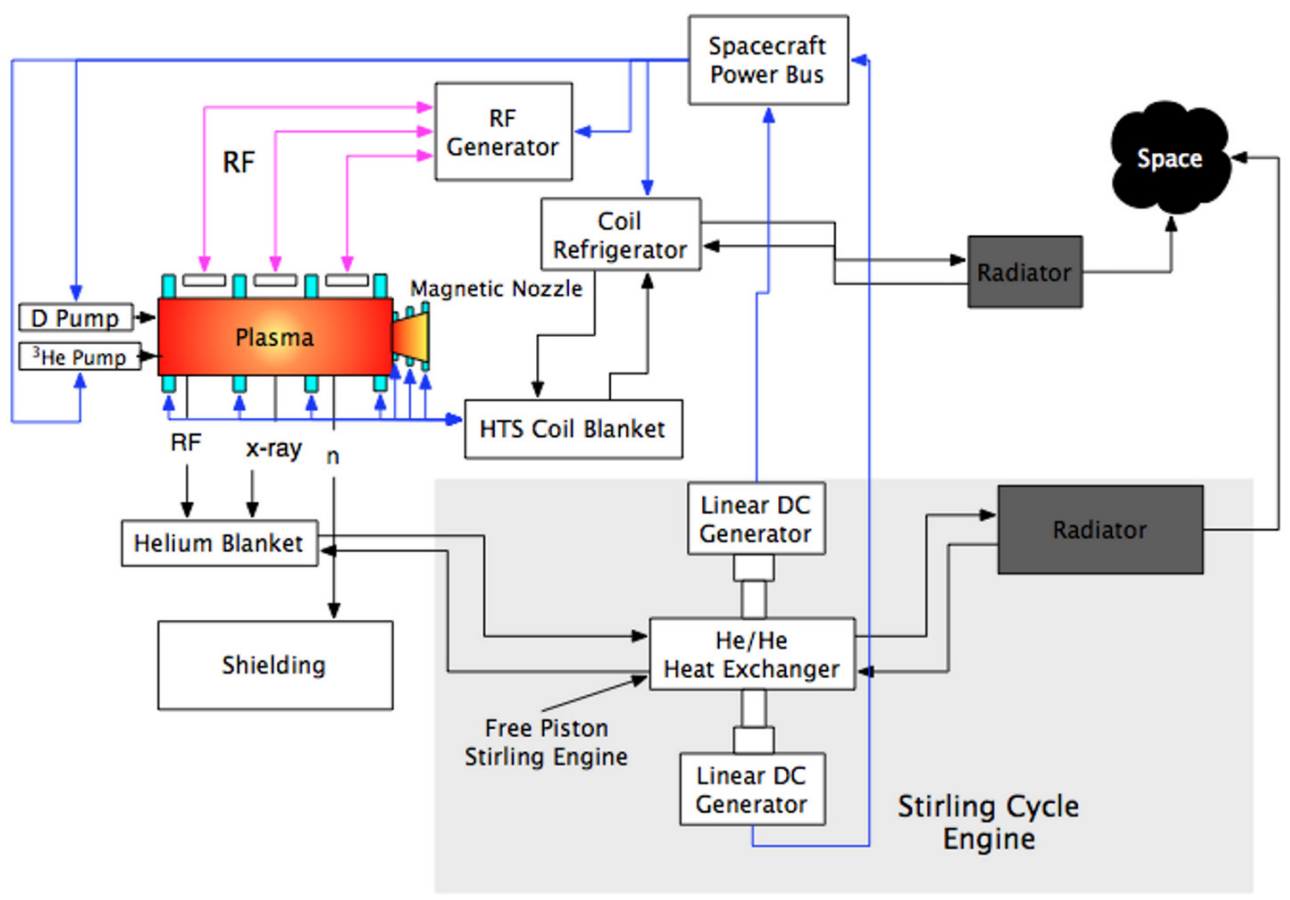

Figure 1: Power balance in an aneutronic fusion propulsion system.

An RF generator heats the plasma, generating the fusion reaction, which releases Bremsstrahlung, synchrotron, and neutron radiation. The neutrons are absorbed by the shielding and the X-rays, by the helium blanket. Heat is converted through a Stirling Cycle engine, where electrical power is recycled back into the craft. Coolant in high-temperature superconducting (HTS) coils absorb some heat. Waste hear from the HTS coils and the thermal power conversion subsystem is rejected to space through radiators.

\section{Fusion Background}

Minimal neutron production is attractive for space propulsion because it reduces the required shielding as well as the engine size, mass, and cost. Additionally, the use of $\mathrm{D}-{ }^{3} \mathrm{He}$ increases the fraction of power available for propulsion by decreasing the energy and quantity of neutrons produced and completely eliminates the need to breed tritium $(\mathrm{T})$. The $\mathrm{p}-{ }^{11} \mathrm{~B}$ aneutronic reaction, though it produces the fewest neutrons of any fusion fuel mixture and has abundantly available fuel, is not considered here because there is strong uncertainty whether net power could be produced and because stronger magnetic fields and higher plasma temperatures would be required. Thus, $\mathrm{D}-{ }^{3} \mathrm{He}$ is the most promising fuel mixture and is the only one considered below.

The $\mathrm{D}-{ }^{3} \mathrm{He}$ plasma admits both $\mathrm{D}-{ }^{3} \mathrm{He}$ and deuterium-deuterium (D-D) reactions. Thus,

$$
\begin{aligned}
\mathrm{D}+{ }^{3} \mathrm{He} & \rightarrow{ }^{4} \mathrm{He}(3.6 \mathrm{MeV})+\mathrm{p}(14.7 \mathrm{MeV}) \\
\mathrm{D}+\mathrm{D} & \rightarrow \mathrm{T}(1.01 \mathrm{MeV})+\mathrm{H}(3.02 \mathrm{MeV}) \\
\mathrm{D}+\mathrm{D} & \rightarrow{ }^{3} \mathrm{He}(0.82 \mathrm{MeV})+\mathrm{n}(2.45 \mathrm{MeV})
\end{aligned}
$$

where the values in parenthesis are the energy of that particular fusion product. The $\mathrm{D}-{ }^{3} \mathrm{He}$ reaction provides a higher power density than the D-D reactions, and both its reaction products are charged, allowing for direct control of the exhaust. For a thermal plasma with equal $\mathrm{D}$ and ${ }^{3} \mathrm{He}$ densities, the fraction of the fusion energy carried by neutrons from the D-D reaction, $f_{\mathrm{p}}$, is $1 / 3$, from which it is difficult to extract useful thrust. If the tritium fusion products of equation (2) also fuse within the plasma, considerably higher fractions of power would be in the undesired neutron channel. To reduce this problematic possibility, two routes have been proposed. The simplest way is to reduce the deuterium fraction in the thermal plasma from the stoichiometric 1:1 mixture, though it would also decrease the power density, if the magnetic field is held constant. Alternatively, [48] and [31] have proposed an $f_{\mathrm{p}}$-reducing method termed T-suppressed D-D fusion (or He-catalyzed D-D) in which the tritium is rapidly removed from the plasma before it can fuse. The tritium that is captured naturally transmutes to ${ }^{3} \mathrm{He}$ after which it is injected back into the plasma as fuel. By this circuitous route, it should be possible to achieve an $f_{\mathrm{p}}$ as low as $5 \%$. However, such a cycle only makes sense for mission durations considerably longer than the 12.3 year half life of tritium. Our RF plasma heating method (see Section 3.2) could reduce $f_{\mathrm{p}}$ to under $0.5 \%$, by tailoring the ion energy distributions, based on [20]. 


\section{Modular Fusion Engine Design}

\subsection{Overview}

The reactor design we propose differs from [16] in size, heating method, and fuel. There, $\mathrm{p}-{ }^{11} \mathrm{~B}$ was selected, which requires five-times higher ion energies and produces far less fusion power per reaction. They also chose neutral beams for heating, requiring a plasma volume that is one hundred times larger, and is therefore more costly and less stable. The even-parity rotating magnetic field $\left(\mathrm{RMF}_{\mathrm{e}}\right)$, see [10] and selected by [38], has been shown to have poor energy confinement, resulting in the need for a larger FRC as well. However, increasing the FRC size to the point where the plasma radius is more than 10 times the ion Larmor radius will lead to magnetohydrodynamic (MHD) instabilities. We selected the odd-parity $\mathrm{RMF}\left(\mathrm{RMF}_{\mathrm{o}}\right)$, instead, which is predicted to promote better energy confinement, hence allow smaller, more stable engines. The overall engine layout is depicted in Figure 1.

Plasma parameters were chosen by an iterative, self-consistent process design around the desired power an estimated energy confinement time (1/5 of classical and radiation losses) and the magnetic field capabilities of current high-temperature superconductors, incorporating a wide safety margin. Further refinements in the plasma modeling was performed to reduce neutron wall load. These included increasing the ${ }^{3} \mathrm{He} / \mathrm{D}$ ratio in the plasma and control of the detailed velocity distributions of the $\mathrm{D}$ and ${ }^{3} \mathrm{He}$. For the selected magnetic field, ca. $5 \mathrm{~T}$, a range of ${ }^{3} \mathrm{He}$ and $\mathrm{D}$ average energies satisfied the mission requirements, with values of $100 \mathrm{keV}$ and $70 \mathrm{keV}$ being typical.

The feasibility of fusion itself has been demonstrated by the Tokamak Fusion Test Reactor (TFTR), the Joint European Torus (JET), and the Japan Torus (JT-60). TFTR has generated a record maximum of 10.7 MW, JET produced 16.1 MW, with a $\mathrm{Q} \approx 0.6$, and JT-60 achieved a $\mathrm{Q} \geq 1.25$ and a record ion temperature of $45 \mathrm{keV}$ (where $\mathrm{Q}$ is the fusion energy gain factor or the ratio of nuclear energy produced to the energy needed to maintain the plasma). Our confidence in the DFD is furthered by the fact that it is far smaller and runs cleaner than the mainline fusion devices, hence improvements in operations can be made far more quickly and at far lower cost.

Many physics challenges remain before the $\mathrm{RMF}_{\mathrm{o}}$-heated $\mathrm{FRC}$ can be developed into a practical reactor. The predictions of excellent energy confinement, stability, efficient electron and ion heating, and current drive to fusionrelevant temperatures must be validated. Substantial progress has occurred in the first three areas. In 2010 and 2012 , TriAlpha Energy Corp reported near-classical energy confinement time in their FRC, [9] and [56]. ${ }^{1}$ Our reactor needs energy confinement time only $1 / 5$ as long as the classical. In 2007, an $\mathrm{RMF}_{\mathrm{o}}$-heated FRC, [22], achieved stable plasma durations 3,000 times longer than predicted by MHD theory ([45]); by 2012 that record was extended to over $10^{5}$ times longer. Finally, theoretical studies by [28], [33], and [23] indicate that $\mathrm{RMF}_{\mathrm{o}}$ will be able to heat plasma electrons and ions to fusion relevant temperatures. These are promising starts, but much research is needed at higher plasma temperatures and densities and with burning, i.e., fusing, plasmas.

\subsection{The $\mathbf{R M F}_{\mathrm{o}}$ Method}

As stated above, for an FRC reactor to burn its D- ${ }^{3} \mathrm{He}$ fuel mixture, the ${ }^{3} \mathrm{He}$ and $\mathrm{D}$ average ion energies must achieve values of around $100 \mathrm{keV}$ and $70 \mathrm{keV}$, respectively. If energetic neutral beam injection were used for achieving this temperatures, the plasma would have to be over $4 \mathrm{~m}$ in diameter in order to "stop" the injected neutral beam. Such a large reactor would produce proportionally large amounts of power, near $1 \mathrm{GW}$. In RF heating methods, on the other hand, power can be absorbed over shorter distances. Using $\mathrm{RMF}_{\mathrm{o}}$ allows the diameter of the plasma to be reduced to a half meter and produce $1 \mathrm{MW}$.

In $\mathrm{RMF}_{\mathrm{o}}$ heating, the maximum ion energy is proportional to the $\mathrm{RMF}_{\mathrm{o}}$ frequency, $\omega_{\mathrm{RMF}}$. Due to a constraint set by the RMF-generated current and the FRC's magnetic field strength, $\omega_{\mathrm{RMF}}$ decreases as the product of a plasma density times the square of the plasma radius. Thus, too large or dense an FRC is not well heated. An optimum FRC for $\mathrm{RMF}_{\mathrm{o}}$ heating of ions to $100 \mathrm{keV}$ and above has a radius in the range $20-30 \mathrm{~cm}$. This places a lower limit on the confinement time required, no worse than a fifth of the classical value, as noted earlier.

Ion heating by $\mathrm{RMF}_{\mathrm{o}}$ is highest near the O-point null line, near the center of the plasma's magnetic axis, where it creates a time-varying azimuthal electric field, [28]. This periodically accelerates ions into betatron orbits and then decelerates them back into cyclotron orbits. Choosing the $\mathrm{RMF}_{\mathrm{o}}$ 's $\omega_{\mathrm{RMF}}$ and amplitude properly allows ions to be

\footnotetext{
${ }^{1}$ Classical confinement time occurs for Coulomb collision driven diffusion only. The confinement time of real plasma is often far less than the classical limit, [57].
} 
pumped up, repeatedly, to energies near the peak in the $\mathrm{D}-{ }^{3} \mathrm{He}$ fusion cross-section and then returned to the bulk temperature. This is a conservative process and satisfies the recirculating energy criterion derived by [44] to sustain, against collisions, a non-Maxwellian distribution that increases the fusion rate and decreases neutron production.

In a $\mathrm{D}-{ }^{3} \mathrm{He}$ plasma, the trajectories of $\mathrm{RMF}_{\mathrm{o}}$-accelerated ions are predicted to form two betatron orbit streams close to the FRC's O-point null line: a D stream and an ${ }^{3} \mathrm{He}$ stream. The deuterium stream ions have half the peak energy of the ${ }^{3} \mathrm{He}$ ions, causing non-zero relative velocity between them. The transverse temperature of each beam is considerably lower than the beam's peak energy, hence deuterium ions collide with each other at a far lower center of mass energy than with ${ }^{3} \mathrm{He}$; accordingly, the D-D neutron production rate falls and $f_{\mathrm{p}}$ is reduced, [20].

\section{Table 3: 1 MW fusion engine.}

Temperatures are approximations for a full non-Maxwellian particle distributions generated and sustained by the $\mathrm{RMF}_{\mathrm{o}}$. The majority of the available electrical power is returned to the $\mathrm{RMF}_{\mathrm{o}}$ to heat the plasma. The vacuum vessel is sized based on the dimensions given below and fashioned from 1/8" stainless steel plating. Heat rejection mass is based on aluminum radiators from [29] and Eq. 10 . The RF power generation mass is based on commercially available units from [55].

\begin{tabular}{|c|c|}
\hline Parameter & Value \\
\hline Beta & 0.88 \\
\hline Plasma radius & $0.25 \mathrm{~m}$ \\
\hline Plasma length & $5.0 \mathrm{~m}$ \\
\hline Axial magnetic field & $4.7 \mathrm{~T}$ \\
\hline Ion temperature & $100.0 \mathrm{keV}$ \\
\hline $\mathrm{e}^{-}$temperature & $300.0 \mathrm{keV}$ \\
\hline Deuterium number density & $5.3 \mathrm{e}+13 \mathrm{~cm}^{-3}$ \\
\hline${ }^{3} \mathrm{He}$ number density & $1.6 \mathrm{e}+14 \mathrm{~cm}^{-3}$ \\
\hline $\mathrm{e}^{-}$number density & $3.7 \mathrm{e}+14 \mathrm{~cm}^{-3}$ \\
\hline \multicolumn{2}{|l|}{ Masses $-\cdots$} \\
\hline$\overline{\text { Vacuum vessel mass }}$ & $800 \mathrm{~kg}$ \\
\hline Magnet mass & $260 \mathrm{~kg}$ \\
\hline RF power generation system mass & $1050 \mathrm{~kg}$ \\
\hline Heat rejection system mass & $385 \mathrm{~kg}$ \\
\hline $\begin{array}{l}\text { Thermal power conversion system } \\
\text { mass }\end{array}$ & 2200 \\
\hline Shielding mass & $1475 \mathrm{~kg}$ \\
\hline Total engine mass & $6170 \mathrm{~kg}$ \\
\hline \multicolumn{2}{|l|}{ Power $(\bar{Q} \approx \overline{2})$} \\
\hline Fusion power & $2.14 \mathrm{MW}$ \\
\hline $\mathrm{RMF}_{\mathrm{o}}$ power & $1 \mathrm{MW}$ \\
\hline Bremsstrahlung power & $490 \mathrm{~kW}$ \\
\hline Synchrotron power & $992 \mathrm{~kW}$ \\
\hline Neutron power & $2.59 \mathrm{~kW}$ \\
\hline Waste heat & $563 \mathrm{~kW}$ \\
\hline Available electrical power & $1.25 \mathrm{MW}$ \\
\hline Propulsion power & $1.13 \mathrm{MW}$ \\
\hline
\end{tabular}

Further reductions in $f_{\mathrm{p}}$ can be gained from the differential in the energy-dependent fusion rates. As can be seen in Figure 2, if the bulk plasma has an average energy of $70 \mathrm{keV}$ and the $\mathrm{RMF}_{\mathrm{o}}$ pumps the ${ }^{3} \mathrm{He}$ up by $100 \mathrm{keV}$ Figure 2, line $a$ then it will pump the deuterium up by only $50 \mathrm{keV}$, Figure 2, line $b$. Thus, several effects-centrally peaked betatron orbits, low transverse beam temperature, reduced D-to- ${ }^{3} \mathrm{He}$ ratio, and higher ${ }^{3} \mathrm{He}$ energy-combine to decrease $f_{\mathrm{p}}$ below $0.2 \%$ for an $\mathrm{RMF}_{\mathrm{o}}$-heated $\mathrm{D}-{ }^{3} \mathrm{He}$-fueled FRC.

Add to these effects that $\mathrm{D}-{ }^{3} \mathrm{He}$ fusion produces neutrons that have only one-sixth of the energy of those produced by burning $\mathrm{D}-\mathrm{T}$ and the larger surface to volume ratio ( $\propto 1 /$ radius) for a small FRC compared to a large tokamak (20 $\mathrm{cm} v$ s. $10 \mathrm{~m}$ ) and an additional 10,000-fold reduction of neutron power load on the wall is obtained. Overall, the 
shielding requirements for this type of small, clean reactor are far less than for a D-T fueled larger fusion engine.

The $\mathrm{RMF}_{\mathrm{o}}$ method, in addition to reducing neutron production, also offers the possibility of a novel, direct energyextraction method from the fusion products. The same rotating azimuthal electric field that heats the ions can be used to extract energy from the $3.6 \mathrm{MeV}$ alphas and $14.7 \mathrm{MeV}$ protons produced by $\mathrm{D}-{ }^{3} \mathrm{He}$ fusion. Depending on phase, a maximum of $2 / 3$ of their energy was seen to be extracted in single-particle Hamiltonian simulations. Inclusion of Coulomb scattering or RMF chirping is expected to increase the number of particles participating in this inverse Landau damping process. The reduction in particle energy is caused by an extraction of energy by the $\mathrm{RMF}_{\mathrm{o}}$ antenna. Thus, $\mathrm{RMF}_{\mathrm{o}}$ could provide both a high-efficiency way of extracting energy directly from the charged fusion products and maintain the center of mass ion energy for the D- ${ }^{3} \mathrm{He}$ collision near the peak of its reactivity, [42].

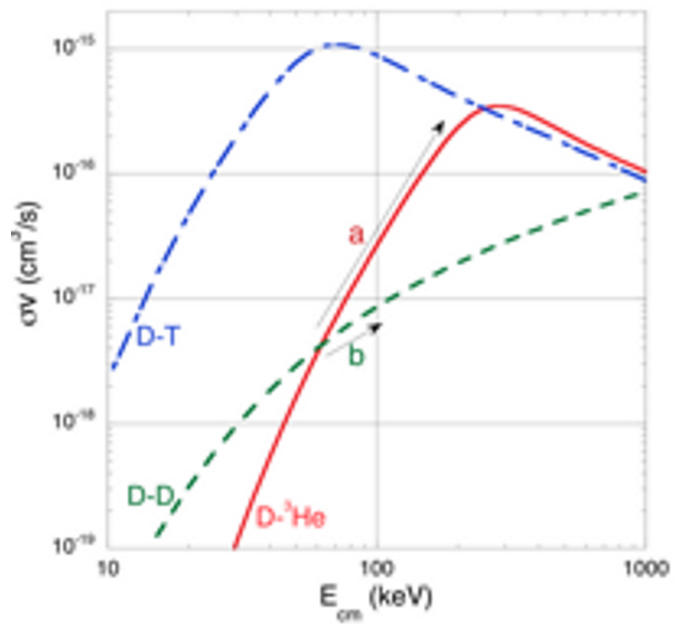

Figure 2: Fusion reaction rates $(\sigma v)$ vs center-of-mass energy $\left(\mathbf{E}_{c m}\right)$ for three sets of collision partners. At ion energies below $250 \mathrm{keV}$, the $\mathrm{D}-{ }^{3} \mathrm{He}$ fusion rate coefficient increases more rapidly with energy than does the DD rate coefficient.

\subsection{One Megawatt Reactor System Design}

The system design is based on on-going research with the Princeton FRC-2 (PFRC-2) and Magnetic Nozzle Experiment (MNX) at the Princeton Plasma Physics Laboratory. The PFRC-2 machine is shown in Figure 3. Based on $1 / 5$ classical confinement time, a plasma radius of $25 \mathrm{~cm}$ is adequate for confining the high energy plasma needed to produce $1 \mathrm{MW}$ of fusion power. This radius matches criteria set by the $\mathrm{RMF}_{\mathrm{o}}$ heating method. Table 3 displays all the design and calculated parameters for the $1 \mathrm{MW}$ reactor, with modest changes in these parameters the fusion power could increase up to $20 \mathrm{MW}$. The plasma is shaped by the magnetic field: a separatrix divides the closedfield region (CFR) from the open-field region (OFR). Field-shaping coils that are magnetic flux conservers surround the plasma. Between the plasma and inner wall of the chamber is a scrape off layer (SOL). Figure 4 illustrates this magnetic field geometry. The chamber wall is composed of a helium cooling blanket for extracting thermal energy from the Bremsstrahlung radiation, neutron shielding, a medium- or high- $Z$ material for X-ray absorption, and structural support. A cross-section of one realization of the reactor walls is depicted in Figure 5.

\subsection{Fuel and Propulsion Subsystem}

The fuel subsystem consists of cryogenic tanks, propellant lines, and a fuel heating system. The small amount of ${ }^{3} \mathrm{He}$ needed for such a mission $(\approx 0.5 \mathrm{~kg}$, see Table 4$)$ is well within current terrestrial production capability. The liquid deuterium and ${ }^{3} \mathrm{He}$ are heated and converted to gases, injected into the FRC (probably as 10-20 keV neutral beams [14]), and heated to fusion temperatures. Deuterium propellant is pumped through the scrape off layer (SOL), where the fusion products rapidly slow down, exhausting the ash (including any of the undesirable $\mathrm{T}$ formed) and heating the SOL, increasing the thrust. 


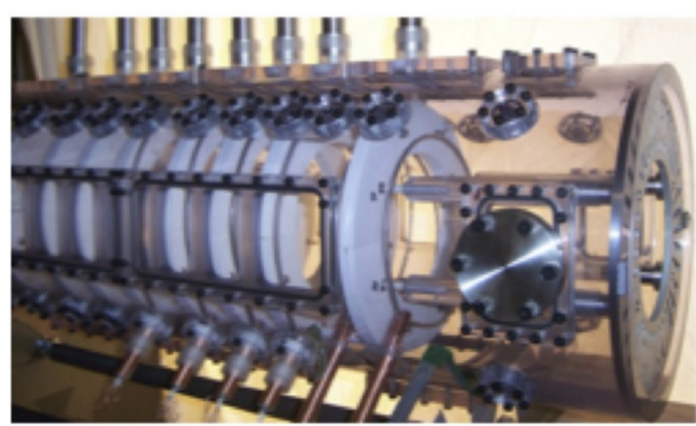

Figure 3: The Princeton Field-Reversed Configuration 2 device at the Princeton Plasma Physics Laboratory. The current machine features a number of field shaping coils (white) that act as flux conservers, positioned axially along the reaction chamber.

\subsection{Startup}

Chemical combustion will be used to produce the power necessary power for starting the reactor. A few kilograms of $\mathrm{H}_{2}$, which produce $142 \mathrm{MJ}$ each when combined with $\mathrm{O}_{2}$, is enough for start up. The power released from this reaction is injected the plasma, energizes the superconducting coils, and heats the plasma through the $\mathrm{RMF}_{\mathrm{o}}$ system. The heat engine and incorporated generator would be used with this additional heat source.

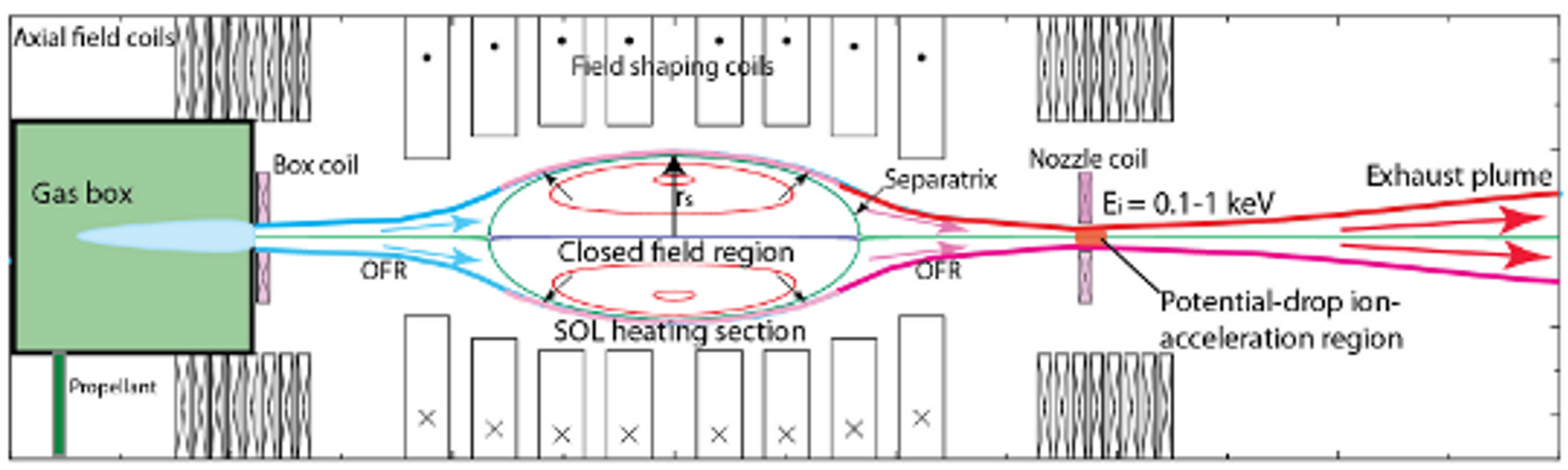

Figure 4: Fusion propulsion system layout.

The magnetic field is shaped by field-shaping coils and axial field coils to form an ellipsoidal closed-field region (CFR) and an open-field region (OFR), between which lies the separatrix. The fusion reaction occurs round the O-point null line, in the CFR, and the products are ejected into the SOL. Propellant is injected into the gas box and flows through the SOL where it gets heated by the fusion products. The heated propellant flows out the nozzle coil and forms an exhaust plume, with ion energy $E_{i}$.

\subsection{RMF Heating}

The $\mathrm{RMF}_{\mathrm{o}}$ heating system generates $\mathrm{RF}$ power proportionally to the ${ }^{3} \mathrm{He}$ cyclotron frequency, such that

$$
\omega_{\mathrm{RMF}}=\alpha \omega_{\mathrm{ic}}=\alpha \frac{Z e B}{m}
$$

where $Z$ is the atomic number, $B$ is the magnetic field, $e$ is the elementary charge, $m$ is the mass of the ion, and $0.01 \leq \alpha \leq 0.1$. Given the axial magnetic field from Table 3, the ion cyclotron frequency for ${ }^{3} \mathrm{He}$ is $61.6 \mathrm{MHz}$ and a $0.6-6 \mathrm{MHz}$ generator will be required.

The RF subsystem consists of the RF generator, coaxial cables, directional couplers, tank circuits, antennas, and RF diagnostics. The RF system must produce enough power to start and sustain the fusion reactions. When the fusion reactor is operating, smaller amounts of RF power are needed because fusion products provide some heating and current drive. The RF generator must produce power with high efficiency; most commercial units operate around 


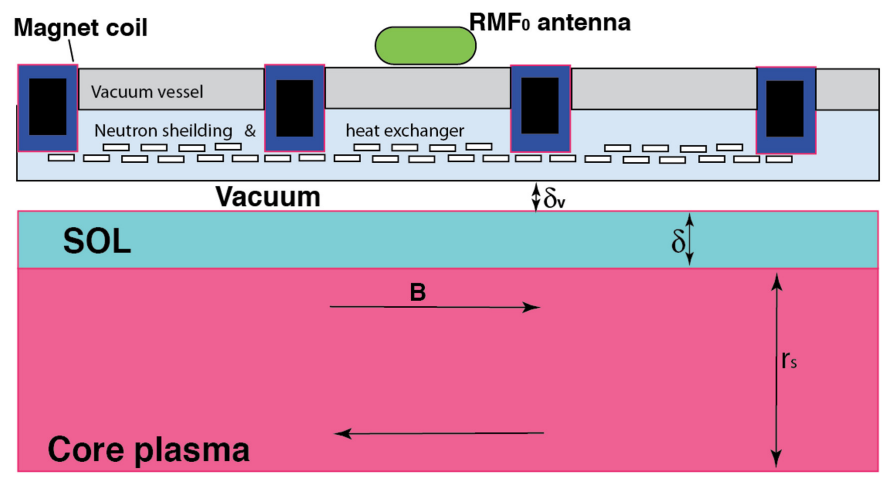

Figure 5: One megawatt reactor cross-section.

The outer edge of the magnetic fields (B) in the core plasma are surrounded by the SOL and a thin vacuum layer. Neutron shielding and the heat exchanger system protect the magnet coils, vacuum vessel, and $\mathrm{RMF}_{\mathrm{o}}$ antenna from radiation damage.

$90 \%$, [55]. This is accomplished by operating the power device as a saturated switch. The PFRC-2 has a $200 \mathrm{~kW}$ RF system and we estimate a peak startup RF power of $2 \mathrm{MW}$ and continuous power of $1 \mathrm{MW}$ will be required for the reactor to produce $2 \mathrm{MW}$ of fusion power.

\subsection{Superconducting Flux Conservers}

Radial confinement is achieved by the coaxial solenoidal field-shaping coil array, commonly termed flux conservers (FCs), which can be seen in Figure 3. FCs are superconducting rings, approximately evenly spaced along the reactor length. The FCs in the PFRC-2 employ high-temperature superconducting materials set in a liquid nitrogen cooled copper mandrel. For a full discussion on the design, construction, and testing of the PFRC-2's FCs, see [39].

\subsection{Reactor Chamber and Shielding}

Even though D- ${ }^{3} \mathrm{He}$ is an "aneutronic" fuel, one of its D-D side reactions produces $2.45 \mathrm{MeV}$ neutrons. As the chamber is subjected to neutron bombardment, it becomes susceptible to several distinct changes in its material properties. The microstructure is altered such that it becomes up to five times stronger in tensile strength when exposed to $\mathrm{MeV}$ neutrons. However, at the same time, this process introduces weakness in the material as it becomes more brittle and prone to cracks and associated damage. Furthermore, the structure also experiences swelling as the atoms are structurally displaced to form voids in the material. In designing the reactor chamber, measures must be taken to promote fracture resistance and accommodate the dimensional alterations that occur given the neutron irradiation, [17]. Many of these shortcomings can be resolved with appropriate shielding. The low- $Z$ material ${ }^{10} \mathrm{~B}_{4} \mathrm{C}$ strongly moderates neutron energies and attenuates their flux by absorption. ${ }^{10} \mathrm{~B}_{4} \mathrm{C}$ is a very sturdy, stable solid over a very large range of temperatures that is readily available in nature, safe to manufacture, inexpensive, and it does not exhibit any obvious structural deformities when irradiated. Since it is transparent to X-rays, Bremsstrahlung radiation can pass through it to a thermal power conversion system. To verify boron carbide numerically, Monte-Carlo simulations were performed assuming isotropic and forward scattering per [8] and using total and absorption cross-section results from MCNP and JEFF3.1[2]. Particles were tracked until reflection or absorption. The simulation found that less than $20 \mathrm{~cm}$ of ${ }^{10} \mathrm{~B}_{4} \mathrm{C}$ shielding would be required to protect the electronics from exposures in excess of one year.

$\mathrm{X}$-ray shielding is also required to absorb stray Bremsstrahlung radiation that is not captured by the thermal power conversion system described in Section 3.8. Tungsten is one of the best attenuators on a per mass basis ([34]) and meets many of the material requirements for shielding including availability, manufacturability, and high temperature capabilities. We calculate that the Bremsstrahlung has a maximum energy of $\approx 150 \mathrm{keV}$ and the transmitted power is given by

$$
I=I_{o} \exp ^{-\alpha t_{m}}
$$

where $I_{o}$ is the initial power, $\alpha$ is the measured attenuation coefficient and $t_{m}$ is the so-called mass thickness, i.e. density $\times$ shield thickness. Tungsten at $150 \mathrm{keV}$ has an $\alpha=1.389 \mathrm{~cm}^{2} / \mathrm{g}$, therefore a million fold reduction in X-ray radiation would require a $0.64 \mathrm{~cm}$ thick shield. [30] 


\section{FRC Coils Throat Coil Nozzle Coils}

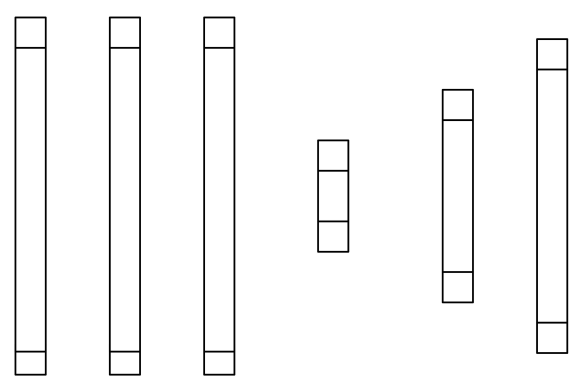

Figure 6: Magnetic nozzle coil configuration.

The throat and nozzle coils allow the magnetic field lines to shape the weakly-ionized propellant into an efficient exhaust plume.

\subsection{Thermal Power Conversion}

As noted above, it is possible to extract energy from the plasma using the RF system. Thermal power from synchrotron and Bremsstrahlung radiation can also be converted to electrical power using a Stirling cycle power generation system, [25]. Studies show that it has the best specific power of all thermal energy conversion systems. Other options are using a Brayton cycle ([36]) and direct conversion methods ([5]). At the temperatures of interest, the Brayton cycle appears to have a lower specific power and direct conversion methods have much lower efficiencies. The Bremsstrahlung can be efficiently removed by flowing helium gas through ${ }^{10} \mathrm{~B}_{4} \mathrm{C}$ heat exchanger tubes containing corrugated sheets of refractory medium $-Z$ material ([24]). The Bremsstrahlung heats the high- $Z$ materials to about $2000 \mathrm{~K}$. A helium coolant extracts this energy and is used to drive a high-efficiency thermal cycle. With a thermal rejection temperature to space of $625 \mathrm{~K}$, this results in a Carnot efficiency, $\eta_{\mathrm{C}}$, of $69 \%$, [19].

\subsection{Magnetic Nozzle and Thrust Augmentation}

The magnetic nozzle and thrust augmentation system allow directional control of the plasma and control of the thrust level and exhaust velocity. A magnetic nozzle, as described by [7], [54], and [21], redirects the flow from the FRC to free space. The nozzle consists of a throat coil and two or more additional nozzle coils to allow expansion and acceleration of the flow. Like the FCs, all the coils are superconducting but the radii of the magnetic nozzle coils are smaller than those of the FC, as can be seen in Figure 6. Magnetic nozzles have been found to be highly efficient, especially with weakly magnetized propellants, with $\eta_{\text {plume }}>85 \%$ in [35].

Thrust is increased by the addition of propellant, increasing mass flow and thrust at the expense of specific impulse, [47]. A suitable propellant would be deuterium, which would be injected as a cold gas into the gas box and from there enter the open-field region. The gas would be ionized quickly, forming a plasma similar to what is called, in tokamak terminology, a high recycling divertor, or even a detached plasma. The cold plasma will flow around the outside of the separatrix with electrons picking up energy from the fusion products and synchrotron radiation as it passes through the OFR. The shell plasma thus formed and heated will be quite similar to that already produced in gas dynamic traps, [6], having a density near $0.1-5 \times 10^{14} \mathrm{per}$ cc and an electron temperature of $30-300 \mathrm{eV}$. The ions will be cooler. As the plasma leaves the reactor, acceleration of the ions to an energy 4-8 times the electron temperature will occur in a double layer near the nozzle throat, [21]. Within a few centimeters beyond the nozzle throat, the propellant will have acquired the desired $0.2-1.2 \mathrm{keV}$ energy. Within the detached plasma regime, UEDGE simulations have shown efficiencies of at least $\eta_{\mathrm{T}}=0.8$ for propellant flow from the gas box to the nozzle for our configuration, [15].

\section{Example Mission}

The DFD's performance was simulated in the context of a mission to deploy the James Webb Space Telescope in a 770,000 km halo orbit around the Sun-Earth L2 point, [27]. The spacecraft acts as a ferry and returns to low earth orbit for restocking and refueling and then can be re-used on further missions, including servicing the telescope. The design parameters of the spacecraft required for this mission are derived from the equations below and are listed in Table 4. 


\section{Table 4: Spacecraft design parameters.}

The payload mass is for the James Webb Space Telescope. $\mathrm{kW}_{\mathrm{e}}$ is the electrical power that needs to be processed by the thermal power conversion system.

\begin{tabular}{|c|c|c|}
\hline Parameter & Symbol & Value \\
\hline Payload Mass & $m_{p}$ & $6200 \mathrm{~kg}$ \\
\hline Total Dry Mass & $m_{d}$ & $12370 \mathrm{~kg}$ \\
\hline Total Fuel \& Propellant Mass & $m_{f}$ & $353 \mathrm{~kg}$ \\
\hline${ }^{3} \mathrm{He}$ Mass & $m_{\mathrm{He}}$ & $0.53 \mathrm{~kg}$ \\
\hline 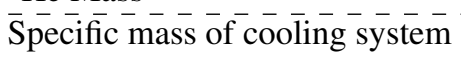 & $\bar{M}_{r}^{-}$ & $-\overline{1.76} \overline{\mathrm{kg}} / \overline{\mathrm{k}} \overline{\mathrm{W}}_{\mathrm{e}}^{-}$ \\
\hline Specific mass of fuel & $\sigma_{f}$ & $0.03 \mathrm{~kg} / \mathrm{kg}$ \\
\hline$\overline{\text { Specific power }}$ & $\alpha_{p}$ & $0.18 \mathrm{k} \overline{\mathrm{W}} / \mathrm{kg}$ \\
\hline Thrust & $T$ & $40 \mathrm{~N}$ \\
\hline Exhaust velocity & $u_{e}$ & $56.5 \mathrm{~km} / \mathrm{s}$ \\
\hline
\end{tabular}

The exhaust stream power required by the spacecraft to make this journey is

$$
P=\frac{1}{2} T u_{\mathrm{e}}
$$

where $P$ is power, $u_{\mathrm{e}}$ is the exhaust velocity, and $T$ is the thrust [43]. As can be seen, given a constant power, the thrust and exhaust velocity can be traded against each other for mission optimization.

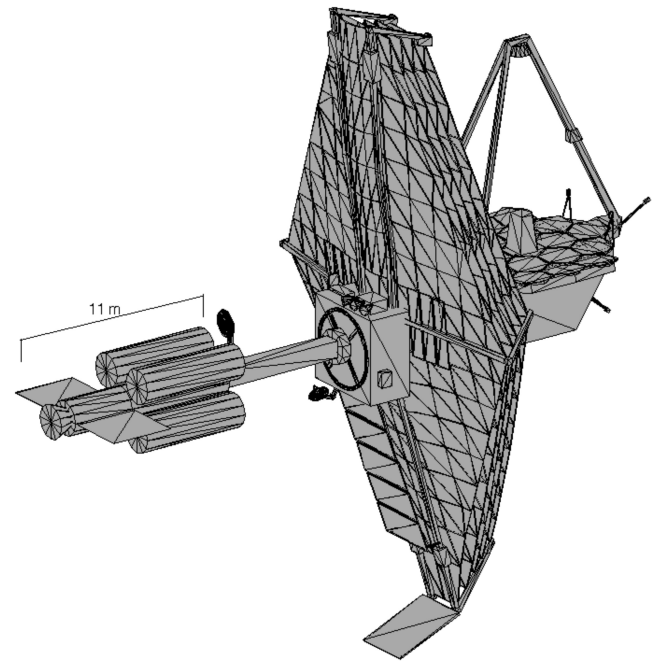

Figure 7: DFD-powered spacecraft carrying the James Webb Space Telescope.

The reactor is attached to the telescope by a pylon. The deuterium tanks, located between engine and the telescope, protect the latter from stray neutron radiation. The total engine length shown includes the propellant tanks.

Trajectory optimization depends on the rocket equation and propellant mass flow to calculate the fuel dynamics. The propellant mass flow is

$$
\dot{m}=\frac{T}{u_{\mathrm{e}}}
$$

or in terms of power

$$
\dot{m}=\frac{2 P}{u_{\mathrm{e}}^{2}}
$$


where $\dot{m}$ is the rate at which mass is exhausted. The rocket equation is

$$
\frac{m_{\mathrm{d}}+m_{\mathrm{f}}}{m_{\mathrm{d}}}=\exp \left(\frac{\Delta u}{u_{\mathrm{e}}}\right)
$$

where $m_{\mathrm{d}}$ is the dry mass, $m_{\mathrm{f}}$ is the mass of the fuel, and $\Delta u$ is the total required velocity change. The dry mass consists of the reactor, payload, and fuel tanks. The fuel tank size can be found iteratively using this equation's solution for fuel mass.

The radiator must be able to reject heat from the non-ejected fusion products, Bremsstrahlung radiation, and neutrons, where

$$
\left.P_{\text {heat }}=\left(1-\eta_{\mathrm{C}}\right)\left(\left(1-\eta_{\mathrm{T}}\right) P_{\text {fusion }}+P_{\text {Bremsstrahlung }}+P_{\text {Synchrotron }}+P_{\text {Neutrons }}\right)\right)
$$

where $P_{x}$ is the power from a given source and $\eta_{\mathrm{C}}$ and $\eta_{\mathrm{T}}$ are, respectively, the Carnot and thrust efficiencies from Section 3. Given the specific mass of the cooling system in Table 4 from [25], the mass of the total cooling system is calculated in Table 3. The radiators can be sized using the Stefan-Boltzman law, an emissivity $(\epsilon)$ of 0.86 as given in [29], with the Stefan-Boltzmann constant $\sigma_{\mathrm{SB}}$, and a rejection temperature, $\mathcal{T}$, of $625 \mathrm{~K}$, the radiator surface area, $A$, is given by

$$
A=\frac{P_{\text {heat }}}{\epsilon \sigma_{\mathrm{SB}} \mathcal{T}^{4}}=77 \mathrm{~m}^{2}
$$

The available power and other key mission parameters are found in Table 4. For any particular total $\Delta u$ there will be an optimal $u_{\mathrm{e}}$ that minimizes the mission mass, which can be easily found from the above equations. A conception of the spacecraft with the JSWT is shown in Figure 7.

An example trajectory is shown in Figure 8. This trajectory is simulated in a non-dimensional, circular restricted three-body problem for the Sun-Earth/Moon system. A baseline delta-v of $3.1 \mathrm{~km} / \mathrm{s}$ is required for a spacecraft initialized in a GPS orbit with low eccentricity, with an additional $3.6 \mathrm{~km} / \mathrm{s}$ required if starting from LEO. Additional work on the trajectory optimization and use of lunar gravity assist may reduce the required delta-v. Thrust is applied for approximately 11 days in a direction normal to the plane defined by the Earth-relative position vector and the orbitnormal vector. This causes the spacecraft to gradually increase the altitude of its Earth orbit as well as its eccentricity, spiraling out until it escapes Earth gravity. This is followed by a coast arc of nearly a week and then a 14 hour insertion burn occurs, decelerating the craft and allowing it to coast into a weakly stable halo orbit about L2 after 37 days. This trajectory was simulated with multiple iterations, adjusting the thrust to control mission duration, and changing the fuel mass and associated dry mass according to equation (8) until the required $\Delta u$ was feasible.

\section{Conclusions}

We have presented the design for the Direct Fusion Drive based on the field-reversed configuration and $\mathrm{RMF}_{\mathrm{o}}$ heating, using D- ${ }^{3} \mathrm{He}$ reaction as an energy source. As an example, we presented a mission from GPS orbit to a Sun-Earth L2 halo orbit. Many of the key physics principles involved have been separately demonstrated. There still remain a number of questions regarding the operation and stability of the reactor, which we hope to answer with future PFRC and MNX experiments. The size, mass ratio, specific power, and thrust make the DFD an attractive option for longer missions, such as to Mars, and crucial for allowing safer and faster manned interplanetary missions. 


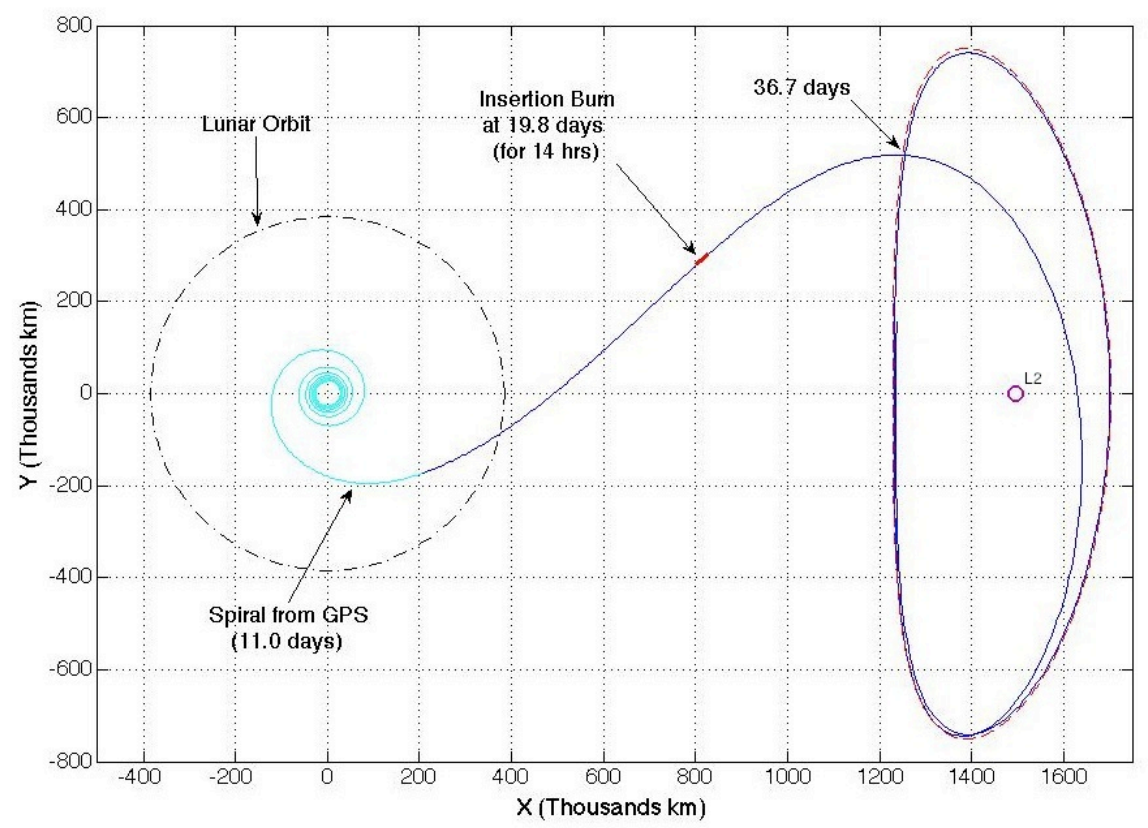

Figure 8: LEO to Sun-Earth L2 halo orbit trajectory for the DFD-powered spacecraft.

This trajectory requires a $\Delta u$ of $3.1 \mathrm{~km} / \mathrm{s}$ from an initial altitude of $20,232 \mathrm{~km}$. If the spacecraft was launched from LEO instead, the mission would require an extra $\Delta u$ of $3.6 \mathrm{~km} / \mathrm{s}$ and 13.8 days for the outbound spiral.

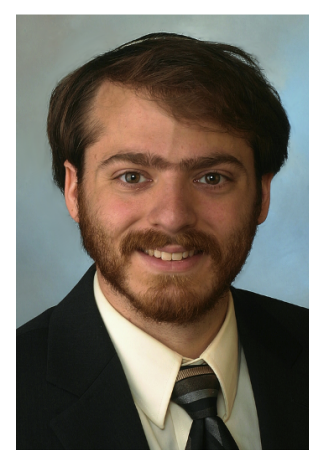

\section{Acknowledgments}

We thank Dr. T. Kornack for calculations on energy extraction by $\mathrm{RMF}_{\mathrm{o}}$. This work was supported, in part, by DOE contract No. DE-AC02-09CH11466.

\section{Vitae}

Yosef S. Razin is a mechanical engineer at Princeton Satellite Systems. He received a BSE in Mechanical and Aerospace Engineering at Princeton University and has worked at the Princeton Plasma Physics Laboratory on the PFRC-2 project. He has also worked at the High-Contrast Imaging Laboratory at Princeton University and the Subaru Telescope in Hilo, HI. His area of interests are clean energy, mechanical design, artificial intelligence, and robotics. He is currently lead engineer on the SunStation, a solar-powered electric vehicle charging station with battery storage and home power back-up. 

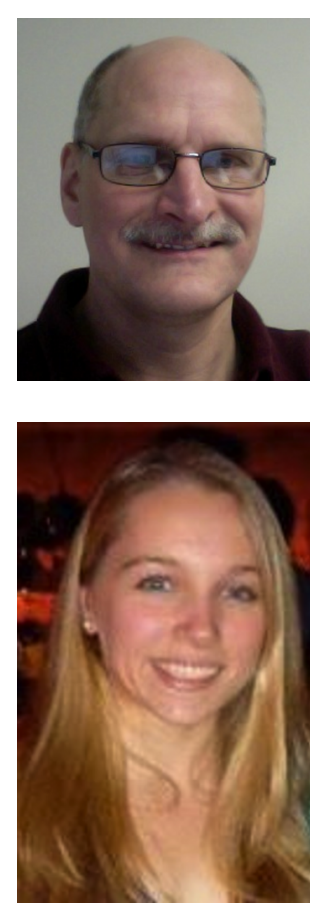

Dr. Gary Pajer is a senior member of the technical staff at Princeton Satellite Systems. He is also Adjunct Professor of Physics at Rider University in Lawrenceville, New Jersey and at The College of New Jersey in Ewing. He received his Ph.D. in solid state physics from the University of Pennsylvania. His area of interest has been in fundamental optical interactions, as well as optical sources and detectors. He has contributed to the development of many devices including spectrometers for research spacecraft, threat detection systems, photonic devices, medical devices, and plasma diagnostic systems.

Mary Breton was an intern at Princeton Satellite Systems and is an undergraduate in the Materials Science and Engineering Department at Massachusetts Institute of Technology. At MIT, she has worked in the PVLab as a member of the Buonassisi Group, producing and characterizing photovoltaic cells with the goal of optimizing the labs firing process. That role is one of many positions she has held that have focused on energy, specifically photovoltaics, in various stages and markets. Once she has a B.S. in Materials Science and Engineering in hand, she envisions being a part of the manufacturing or industrial engineering industries.

Eric Ham was an an intern at Princeton Satellite Systems and is a sophomore at Princeton High School.

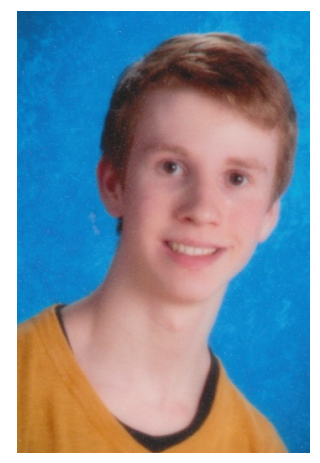



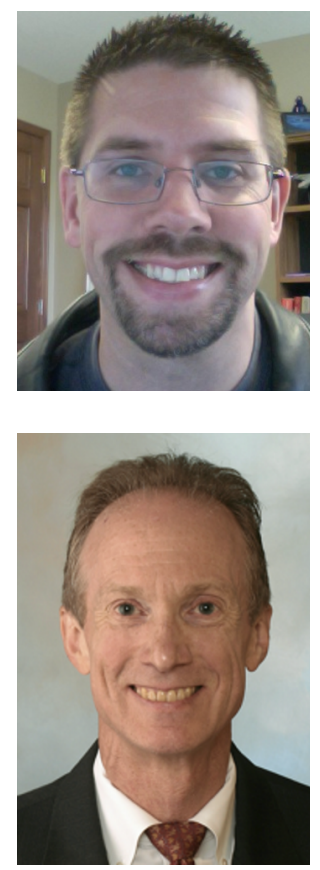

Joseph Mueller graduated from the University of Minnesota with a B.S., M.S. and Ph.D. in Aerospace Engineering. He joined Princeton Satellite Systems in 2000, where he has worked in the areas of spacecraft autonomy, formation flying, high altitude airships, optimization and decision support. He led the design of the Prisma safe orbit guidance system and has been the principal investigator for numerous Small Business Innovative Research (SBIR) contracts with NASA and DoD. He is currently leading a Navy SBIR, developing a decision support framework that generates multiple alternative courses of action for satellites and other complex systems. Joe lives in Minneapolis with his wife and three children.

Michael Paluszek is President of Princeton Satellite Systems He received an EAA and SM in Aeronautics and Astronautics, Massachusetts Institute of Technology in 1979, and an SB in Electrical Engineering from MIT in 1976. He has worked on the PFRC fusion project and on a Tokamak-based fusion rocket both with PPPL. He designed the Attitude Control System for the OSC Indostar-1 satellite and led the effort to develop the TDRS momentum management system for Hughes. Prior to founding Princeton Satellite Systems in 1992, Mr. Paluszek worked at GE Astro Space and the Charles Stark Draper Laboratory.

Alan H. Glasser is a Senior Principal Research Scientist in the Department of Aeronautics and Astronautics at the University of Washington in Seattle. He received a BA in Physics at Columbia College in New York and an M.S. and Ph.D. in Physics at the University of California at San Diego. He has been a research staff member at the Princeton

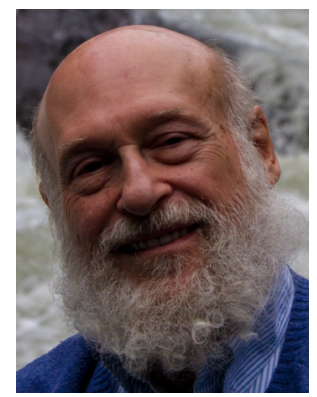




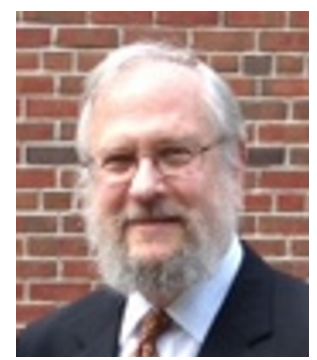

Plasma Physics Laboratory, a Professor of Physics at Auburn University, and a technical staff member and group leader at Los Alamos National Laboratory. He is a Fellow of the American Physical Society. He is internationally known for his work in theoretical and computational plasma physics with application to magnetic fusion energy, including magnetohydrodynamic stability and dynamic modeling of fusion plasmas and complex particle orbits.

Samuel Cohen (B.S./Ph.D. Physics, MIT) is a lecturer with the rank of professor in the Department of Astrophysical Sciences at Princeton University and associated faculty in the Princeton Institute for the Science and Technology of Materials and the Princeton Environmental Institute. He serves as co-director of the Program in Plasma Science and Technology, School of Engineering and Applied Science and is Principal Research Physicist at the Plasma Physics Laboratory (PPPL). His research interests are in the physics of field-reversed configuration plasmas, with emphasis on fusion issues. His honors include: Telluride Scholar, General Motors Scholar, and Goodwin Medal for Distinguished Teaching.

\section{References}

[1] R1-10a-4-2.

[2] Table of nuclides. Korea Atomic Energy Research Institute, 2000.

[3] Technology, 2013.

[4] M. Andrenucci, F. Paganucci, and P. Rossetti. New developments in applied field mpd thrusters. Number IWCP-43-1, Pozzuoli, Italy, June 2002.

[5] J. A. Angelo and D. Buden. Space Nuclear Power. Orbit Book Company, 1985.

[6] A. Anikeev, P. Bagryansky, A. Ivanov, A. Karpushov, K. Noack, and S. Strogalova. Upgrade of the Gas Dynamic Trap: Physical Concepts and Numerical Models. In 28th EPS Conf. on Control. Fusion and Plasma Phys., volume ECA, 25A, page 121, 2001.

[7] A. V. Arefiev and B. N. Breizman. MHD Scenario of Plasma Detachment in a Magnetic Nozzle. Technical report, Inst. for Fusion Stud., Univ. of Tex., July 2004.

[8] A. F. Bielajew. Fundamentals of the Monte Carlo Method for Neutral and Charged Particle Transport. The University of Michigan, http://www-personal.umich.edu/ bielajew/MCBook/book.pdf, September 2001.

[9] M. Binderbauer, H. Y. Guo, M.Tuszewski, S. Putvinski, L. Sevier, and D. Barnes. Dynamic Formation of a Hot Field Reversed Configuration with Improved Confinement by Supersonic Merging of Two Colliding High-Compact Toroids. Phys. Rev. Lett., 105:045003, 2010.

[10] H. Blevin and P. Thonemann. Nucl. Fusion Suppl., 1:55, 1962.

[11] C. Bramanti, D. Izzo, T. Samaraee, R. Walker, and D. Fearn. Very High Delta-V Missions to the Edge of the Solar System and Beyond Enabled by the Dual-Stage 4-Grid Ion Thruster Concept. Acta Astronautica, 64(78):735-744, 2009.

[12] C. Bramanti, R. Walker, O. Sutherland, R. Boswell, C. Charles, P. Frigot, M. Orlandi, J. del Amo, and D. Fearn. The Innovative Dual-Stage 4-Grid Ion Thruster Concept-Theory And First Experimental Results. Number IAC-06-C4. 4.7, Valencia, Spain, 2006. 57th Int. Astronautical Congr.

[13] Busek. Hall Effect Thrusters, 2012.

[14] M. Buttolph. Neutral beam presentation. PPPL SULI Program, 2013.

[15] M. C. Cheong. Analysis of Heat and Particle Flows in the Scrape-Off Layer of a Field-Reversed Configuration. 2012 Internsh. Program, Program in Plasma Sci. \& Tech., PPPL, October 2012.

[16] A. Cheung, M. Binderbauer, F. Liu, A. Qerushi, N. Rostoker, and F. J. Wessel. Colliding Beam Fusion Reactor Space Propulsion System. In Space Tech. and Appl. Int. Forum, number CP699, 2004.

[17] O. K. Chopra. Degradation of LWR Core Internal Materials due to Neutron Irradiation. Technical Report NUREG/CR-7027, Argonne National Laboratory, December 2010.

[18] J. W. Clark and G. H. McLafferty. Summary of research on the nuclear light bulb reactor. Technical report, United Aircraft Reseacrh Laboratories, 1970.

[19] S. Cohen. A Fusion Power Plant Without Plasma-Material Interactions. Technical Report PPPL-3245, Princeton Plasma Physics Laboratory, September 1997.

[20] S. Cohen and C. Liu. Private communication. 2012. 
[21] S. Cohen, X. Sun, N. Ferraro, E. Scime, M. Miah, S. Stange, N. Siefert, and R. Boivin. On Collisionless Ion and Electron Populations in the Magnetic Nozzle Experiment (MNX). IEEE Trans. on Plasma Sci., 34(3):792-803, june 2006.

[22] S. A. Cohen, B. Berlinger, C. Brunkhorst, A. Brooks, N. Ferarro, D. Lundberg, A. Roach, and A. Glasser. Formation of Collisionless High- $\beta$ Plasmas by Odd-Parity Rotating Magnetic Fields. Phys. Rev. Lett., 98:145002, 2007.

[23] S. A. Cohen, A. S. Landsman, and A. Glasser. Stochastic Ion Heating in a Field-Reversed Configuration Geometry by Rotating Magnetic Fields. Phys. of Plasmas, 14:072508, 2007.

[24] J. Dawson. Advanced Fusion Reactors, volume B, page 453. Academic Press, New York, 1981.

[25] M. Dhar. Stirling Space Engine Program. Technical Report NASA/CR-1999-209164/VOL, Glenn Research Center, August 1999.

[26] K. M. Edmondson, D. Law, G. Glenn, A. Paredes, R. R. King, and N. H. Karam. Flexible III-V Multijunciton Solar Blanket. Technical report, Spectrolab, Inc, 2006.

[27] J. P. Gardner and et al. James Webb Space Telescope. Space Sci. Rev., 123:485-606, 2006.

[28] A. Glasser and S. A. Cohen. Ion and Electron Acceleration in the Field-Reversed Configuration with an Odd-Parity Rotating Magnetic Field. Phys. of Plasmas, pages 2093-2102, 2002.

[29] A. Hertzberg. Space Resources: Energy, Power, and Transport, volume 2, chapter Thermal Management in Space. NASA, 1992.

[30] J. H. Hubbell and S. M. Seltzer. Tables of x-ray mass attenuation coefficients and mass energy-absorption coefficients from 1 kev to 20 mev for elements $\mathrm{z}=1$ to 92 and 48 additional substances of dosimetric interest, 1996. Private Correspondence.

[31] V. I. Khvesyuk, N. V. Shabrov, and A. N. Lyakhov. Ash Pumping From Mirror and Toroidal Magnetic Confinement Systems. Fusion Tech., 27:406-408, 1995.

[32] D. Landau and N. Strange. Near-Earth Asteroids Accessible to Human Exploration with High-Power Electric Propulsion. AAS/AIAA Astrodyn Specialist Conf., 11(446), 2011.

[33] A. S. Landsman, S. A. Cohen, and A. Glasser. Onset and Saturation of Ion Heating by Odd-Parity Rotating Magnetic Fields in an FRC. Phys. Rev. Lett., 96:015002, 2006.

[34] R. Lewis. Space Shielding Materials for Prometheus Application. Technical Report MDO-723-0049, Knolls Atomic Power Laboratory, January 2006.

[35] M. M. Martinez. On Plasma Detachment in Propulsive Magnetic Nozzles. Phys. of Plasmas, 18(035504), 2011.

[36] L. S. Mason. A Power Conversion Concept for the Jupiter Icy Moons Orbiter. Technical Report NASA/TM?2003-212596, Glenn Research Center, September 2003.

[37] G. McLafferty and H. E. Bauer. Studies of Specific Nuclear Light Bulb and Open-Cycle Vortex-Stabilized Gaseous Nuclear Rocket Engines. Technical report, NASA, 1968.

[38] K. Miller, J. Slough, and A. Hoffman. An Overview of the Star Thrust Experiment. In Space Tech. and Appl. Int. Forum, volume 420, pages 1352-1358, http://depts.washington.edu/rppl/programs/stx.pdf, 1998. AIP Conference Proceedings.

[39] C. E. Myers, M. R. Edwards, B. Berlinger, A. Brooks, and S. A. Cohen. Passive Superconducting Flux Conservers for Rotating-MagneticField Driven Field-Reversed Configurations. Fusion Sci. E Tech., 61(4587):86, January 2012.

[40] NASA Glenn Research Center. NEXT Ion Propulsion System Development Status and Capabilities, number 14, Cleveland, Ohio, 2007. NSTC.

[41] F. Paganucci, P. Rossetti, and M. Andrenucci. Performance of an Applied Field MOD Thruster. In 27th IEPC, 01-132, Pasadena, CA, October 2001. Electric Propuls. Soc., Centrospazio.

[42] G. Pajer, Y. Razin, M. Paluszek, A. Glasser, and S. A. Cohen. Modular Aneutronic Fusion Engine. In Space Propuls. 2012. AAF-EAS-CNES, 2012.

[43] M. Paluszek, Y. Razin, G. Pajer, J. Mueller, and S. J. Thomas. Spacecraft Attitude and Orbit Control, volume 1, chapter 11: Propulsion. Princeton Satellite Systems, Inc, 3rd edition, 2012.

[44] T. Rider. Fundamental Limitations on Plasma Fusion Systems Not in Thermodynamic Equilibrium. Phys. of Plasmas, 4:1039, 1997.

[45] M. N. Rosenbluth and M. N. Bussac. MHD Stability of Spheromak. Nucl. Fusion, 19:489, 1978.

[46] P. Rossetti, C. Casaregola, and M. Andrenucci. 30kw-class hall thruster: a key building block for propulsion needs of future space transportation and exploration. In IEPC-2011, number 211, Wiesbaden, Germany, September 2011. IEPC.

[47] J. F. Santarius. Lunar ${ }^{3}$ He, Fusion Propulsion, and Space Development. In W. W. Mendell, J. W. Alred, L. S. Bell, M. J. Cintala, T. M. Crabb, R. H. Durrett, B. R. Finney, H. A. Franklin, J. R. French, \& J. S. Greenberg, editor, Lunar Bases and Space Activities of the 21 st Century, pages 75-81, Sept. 1992.

[48] M. Sawan, S. Zinkle, and J. Sheffield. Impact of Tritium Removal and ${ }^{3}$ He Recycling on Structure Damage Parameters in a D-D Fusion System. Fusion Eng. and Des., 61-62:561-567, November 2002.

[49] B. G. Schnitzler, S. K. Borowski, and J. E. Fittje. 25,000-lbf Thrust Engine Options Based on the Small Nuclear Rocket Engine Design. In AIAA, editor, 45th AIAA/ASME/SAE/ASEE Jt. Propuls. Conf. E Exhib, volume 5239. AIAA, 2009.

[50] D. Shiga. Next-Generation Ion Engine Sets New Thrust Record, 2007.

[51] Space and Tech. RL-10 Specifications, 2012.

[52] J. Staff. Prometheus project final report. Technical Report 982-R120461, Jet Propulsion Laboratory, October 2005.

[53] M. Tajmar. Advanced space propulsion systems.

[54] A. G. Tarditi and L. C. Steinhauer. Plasma Flow Control in a Magnetic Nozzle for Electric Propulsion and Fusion Scrape-Off Layer Applications. Number 2C31. 2011 Int. Sherwood Fusion Theory Conf., November 2011.

[55] TEKNA. 1 MW Industrial Unit, 2012.

[56] M. Tuszewski, A. Smirnov, and M. Thompson. Field Reversed Configuration Experiment Through Edge-Biasing and Neutral Beam Injection. Phys. Rev. Lett., 108(255008), June 2012.

[57] J. Wesson. Tokamaks. Oxford Univ. Press, Oxford, 3 edition, 2004. 